\title{
Post-operative analgesia for Oesophagectomy - Thoracic epidural or paravertebral blockade?
}

\author{
Nair A, Lyons IS, Veitch J Parsons S, Carney A \\ Nottingham University Hospitals, City Hospital Campus, Hucknall Road, Nottingham, \\ NG5 1PB
}

Introduction: Oesophagectomy is the only curative treatment for oesophageal cancer. Surgical access for oesophagectomy may be gained via a single thoracoabdominal (TA) incision or separate thoracic and abdominal incisions (ILO). The goldstandard analgesia has been seen as the Thoracic epidural (TE), however this is time consuming to insert, may be uncomfortable to the patient, and may cause significant adverse events. In light of recent work suggesting that paravertebral blockade (PVB) produced a comparable analgesia, and a superior side-effect profile in major thoracic surgery ${ }^{2}$ we assessed the efficacy of PVB and TE for oesophagectomy.

Methods: 154 consecutive patients undergoing oesophagectomy between July 2012 and August 2014 were reviewed. Patient demographics, modality of analgesia and surgical approach were noted. Pain scores in recovery and for 3 days post-op were also recorded. Pain scores were recorded on a scale of $0-3$, where severe pain was 3 , whilst no pain was 0 . in 6 cases there was insufficient data for analysis.

Results: $59(38.3 \%)$ cases underwent ILO, 73 (47.4\%) underwent thoracoabdominal oesophagectomy. The remainder received McKeown's three-stage) oesophagectomy (18 cases; $11.7 \%$ ) or Lap-assisted ILO (17 cases; 11\%).

$66 \%$ of patients undergoing ILO received Thoracic epidural for post-op analgesia, $18.5 \%$ received Paravertebral blockade. No significant difference was noted in the level of analgesia postoperatively; both groups reported no/mild discomfort in more than $85 \%$ of cases Fig 1a).

59 patients $(80.8 \%)$ of those undergoing TA oesophagectomy received thoracic epidurals and 11 (15.1\%) received paravertebral blockade; in 3 cases, other combinations of analgesia were used. In recovery and on days 1 and 2 postoperatively, a greater proportion of patients receiving paravertebral blockade reported moderate or severe pain (Fig 1b).
Discussion: Whilst Thoracic epidural remains the goldstandard for post-operative analgesia in many cases, it is not without its drawbacks. For the ILO, paravertebral blockade provides similar levels of analgesia and are less timeconsuming to place (and are placed under direct surgical vision, whilst the patients is anaesthetised) as well as having less impact on cardiovascular stability.

In the case of Thoracoabdominal Oesophagectomy, Paravertebral blockade would appear to be less effective that Thoracic epidural.

As such we have adopted Paravertebral blockade as our favored modality of analgesia following ILO, to improve patient experience in the context of enhanced recovery.

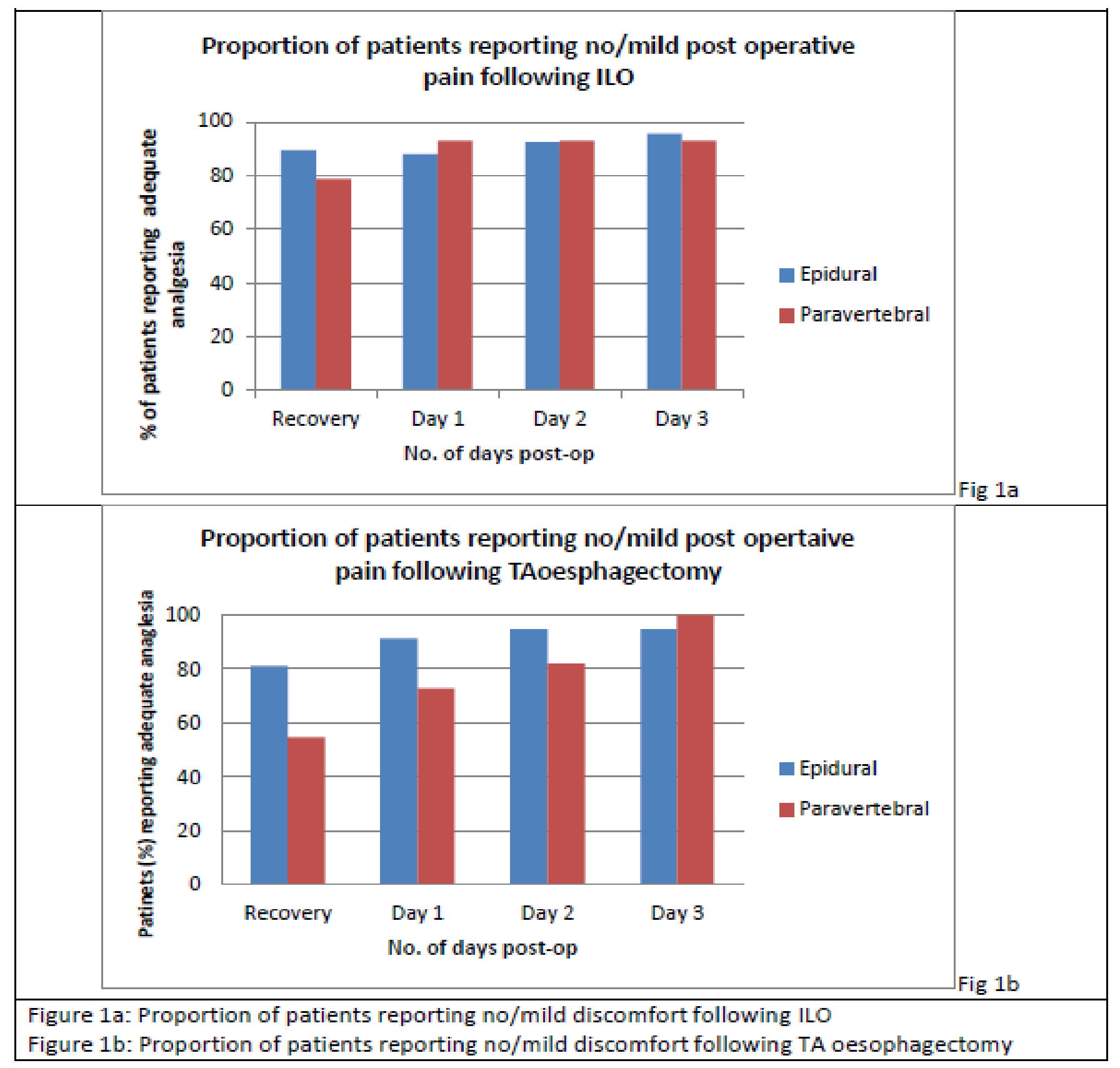

\section{References:}

1. Leslie K, Myles $\mathrm{P}$ et al Neuraxial block, death and serious cardiovascular morbidity in the POISE trial 'Br J Anaesth. (2013) 111(3):382-390

2. Davies RG Myles PS et al 'A comparison of the analgesic efficacy and side effects of paravertebral vs epidural blockade for thoracotomy' Br J Anaesth (2006) 90 (4):418-426y 